\title{
Qualidade de Vida de Estudantes de Medicina medida pelo WHOQOL-bref - UERJ, 2010
}

\author{
Quality of Life of Medical Students by \\ WHOQOL-bref scoring - UERJ, 2010
}

Ana Claudia Santos Chazan Mônica Rodrigues Campos ${ }^{I I}$

\section{PALAVRAS-CHAVE: \\ - Qualidade de Vida; \\ - Educação de Graduação em Medicina; \\ - Estudantes de Medicina; \\ - Doença Crônica.}

\section{KEYWORDS:}

- Quality of Life;

- Education, Medical,

Undergraduate;

- Students, Medical;

- Chronic Disease.

Recebido em: 21/08/2012

Reencaminhado em: 10/10/2012

Reencaminhado em: 0404/2013

Reencaminhado em: 26/05/2013

Aprovado em: $31 / 07 / 2013$

REVISTA BRASILEIRA DE EDUCAÇÃO MÉDICA $376 \frac{137(3): 376-384 ; 2013}{30}$

\footnotetext{
I Universidade do Estado do Rio de Janeiro, Rio de Janeiro, RJ, Brasil.

II Fundação Oswaldo Cruz,Rio de Janeiro, RJ, Brasil.

\section{RESUMO}

O WHOQOL-bref foi utilizado em estudo transversal para avaliar a influência do sexo, da forma de ingresso, do ano de graduação, da classe econômica e da presença de morbidade crônica referida (MCR), na qualidade de vida (QV) de 394 estudantes de medicina da UERJ (72\% dos 545 matriculados em 2010, erro 6,5\%). A análise estatística foi feita no SPSS v17 e utilizados testes qui-quadrado, ANOVA/ pós Hoc de Bonferroni ( p valor $=5 \%$ ) e razão dos produtos cruzados ("Odds Ratio" - OR) com respectivos intervalos de confiança (IC 95\%). A idade média foi de 23 anos, sendo $61 \%$ mulheres, $43 \%$ cotistas (dos quais $64 \%$ mulheres) e $20 \%$ referiu morbidade crônica. De 0-100, a média da QV referida foi 66 e da satisfação com saúde 62. Os menores escores de QV foram observados nos estudantes do sexo feminino, com morbidade crônica referida (MCR), que ingressaram por reserva de vagas, da classe $C$ e do terceiro e sexto ano. Os resultados apontam para a necessidade de estudos qualitativos para aprofundar as informações sobre a $\mathrm{QV}$ dos estudantes da UERJ.

WHOQOL-bref scoring was used in this cross-sectional study to assess the influence of sex, form of admission, year of graduation, economic class and the presence of referred chronic morbidity (RCM) on the quality of life (QOL) of 394 medical students from UERJ (72\% of 545 enrolled in 2010, margin of error 6.5\%). The statistical analysis was performed in SPSS v17 using chi-squared tests, Bonferroni post-hoc ANOVA (pvalue=5\%) and odds ratio (OR) with corresponding confidence intervals (CI 95\%). Mean age was 23 years, with 61\% women, $43 \%$ admitted under positive discrimination ethnic quotas (64\% women) and 20\% referred chronic disease. From 0 to 100, the average referred QOL was 66 and satisfaction with health was 62. The lowest QOL scores were observed among $3^{\text {rd }}$ and $6^{\text {th }}$ year students who were women, with RCM, who had been admitted via reserved university places and belonged to economic class $C$. The results indicate the need for qualitative studies to improve our understanding of the QOL of UERJ students. 


\section{INTRODUÇÃO}

O conceito de qualidade de vida (QV) começou a ser desenvolvido em meados da década de setenta a partir de uma convergência de interesses que surgiram no campo das ciências sociais, econômicas, humanas e da saúde ${ }^{1}$. No âmbito da saúde, destacam-se a insuficiência do conhecimento biomédico para aliviar o sofrimento das pessoas nas situações onde a cura não é possível ${ }^{2}$ e a valorização da relação médico-paciente para o sucesso das intervenções terapêuticas ${ }^{3}$.

São grandes os esforços feitos desde então para o aprimoramento de modelos teóricos sobre $\mathrm{QV}^{1,4}$ e para a construção de instrumentos de fácil aplicação que sintetizem valores de indivíduos e coletividades, a despeito das diferenças sociais e culturais existentes ${ }^{5}$. A Organização Mundial de Saúde (World Health Organization - WHO), a partir de um esforço cooperativo de alguns pesquisadores de várias partes do mundo, criou o Grupo de Qualidade de Vida (Quality of life - QOL) The WHOQOL Group ${ }^{6}$, que define QV como sendo "a percepção do indivíduo de sua posição na vida no contexto da cultura e sistema de valores nos quais ele vive e em relação aos seus objetivos, expectativas, padrões e preocupações".

Os instrumentos então desenvolvidos por esta organização e já validados no Brasil para o português - WHOQOL $100^{7}$ (instrumento com 100 questões sobre QV) e WHOQOL-bref ${ }^{8}$ (versão abreviada com 26 questões) — baseiam-se nos pressupostos de que QV é uma construção subjetiva, só possível de ser avaliada pelo próprio sujeito, multidimensional e composta por elementos positivos (que devem estar presentes, como a mobilidade) e negativos (que devem estar ausentes, como a dor).

As questões relacionadas ao domínio físico abrangem aspectos como a presença de dor e desconforto, energia e fadiga, sono e repouso, mobilidade, atividades da vida cotidiana, dependência de medicação ou de tratamentos e capacidade de trabalho, aspectos que se relacionam à capacidade física. Entretanto, esse instrumento não tem como objetivo uma descrição do estado da saúde funcional, mas do modo de perceber e reagir aos muitos aspectos e domínios da vida, que vão além da saúde física, aproximando-se de um conceito mais ampliado de saúde. Assim, a vida em família, trabalho, moradia, segurança, rede de apoio social e finanças são aspectos da experiência humana com um peso alto para a $\mathrm{QV}$, considerada hoje um desfecho em saúde no campo da promoção.

Sobre o uso do WHOQOL-bref como instrumento de coleta de dados para avaliações de QV, um estudo de revisão revelou que $80 \%$ dos 169 artigos publicados de 1998 a 2006 estão concentrados a partir de 2004, numa tendência crescente, em que o Brasil se destaca como o país que mais publicou em comparação aos outros 33 países representantes de todos os continentes. Além disso, 23\% dos estudos dessa amostra foram realizados em população geral, e $20 \%$ abordaram a QV em um grupo com a análise comparativa de subgrupos?

Nos últimos anos, esse instrumento vem sendo aplicado também em nosso meio para a realização de pesquisas sobre a QV dos estudantes de medicina ${ }^{10,11,12,13}$, sendo que, recentemente, um estudo da Nova Zelândia detectou adequada consistência interna com alfa de Cronbach de 0,89 para a qualidade de vida aferida ${ }^{14}$ de uma amostra de estudantes de medicina.

O objetivo deste estudo é conhecer como o sexo, a forma de ingresso na faculdade, a presença de morbidade crônica referida, o ano de graduação e a classe econômica (variáveis de exposição para efeito da análise estatística) influenciam a QV do estudante de medicina da Faculdade de Ciências Médicas da Universidade do Estado do Rio de Janeiro (FCM/UERJ), de modo a contribuir com elementos para a reflexão dos docentes e discentes sobre o tema.

\section{METODOLOGIA}

Trata-se de estudo exploratório descritivo transversal de uma amostra representativa por período anual de graduação, composta de 394 estudantes (erro de 6,5\%), que representam $72 \%$ dos matriculados na FCM/UERJ em 2010.

Aplicou-se o instrumento WHOQOL-bref adaptado ${ }^{15}$, que contém 26 questões, das quais as duas primeiras são sobre a autoavaliação do entrevistado sobre sua QV (qualidade de vida referida - QVR) e sobre sua satisfação com a saúde (SS). As demais 24 questões são distribuídas em quatro domínios: físico (sete questões), psicológico (seis), relações sociais (três), meio ambiente (oito). Assim, os domínios são representados por 24 facetas, e cada faceta é composta por uma pergunta9 . Todas as questões foram formuladas para respostas do tipo Likert, com escala de intensidade (nada/extremamente), capacidade (nada/completamente), frequência (nunca/sempre) e avaliação (muito insatisfeito/muito satisfeito; muito ruim/ muito bom). Seu autopreenchimento leva cerca de dez a quinze minutos ${ }^{8}$. Os escores são transformados em uma escala linear que varia de $0-100$, sendo estes respectivamente os valores menos e mais favoráveis de QV.

Foram acrescentadas ao WHOQOL-bref questões para identificação do sexo, da idade, do ano da graduação em curso, da renda familiar aproximada em reais e do número de pessoas além do estudante que dependem dessa renda, da forma de ingresso na faculdade (por cota ou não), da classificação econômica pelo critério Brasil $2008^{16}$ e do grau de instrução do chefe da família. Além disso, realizou-se a investigação sobre a presença de morbidade crônica referida (MCR) por meio da 
pergunta: "Você faz tratamento continuado para alguma doença? Em caso afirmativo, que doença?", sendo os resultados apresentados segundo os capítulos da Classificação Internacional das Doenças (CID-10).

A coleta de dados foi realizada nos meses de abril e maio de 2010, e a entrada dos dados foi feita com dupla digitação, conferência cruzada e máscara eletrônica Access ${ }^{\circledR}$, com critério de controle de qualidade, e a base final analisada no SPSS v.17. Para a análise de dados foram utilizados testes qui-quadrado, ANOVA/pós Hoc de Bonferroni e razão de produtos cruzados ("Odds Ratio" - OR), com seus respectivos intervalos de confiança (IC 95\%), para a análise estatística da detecção de diferenças entre proporções/médias (p-valor $5 \%$ ), entre os estratos: sexo (masculino/feminino), MCR (sim/não), forma de ingresso por cota (sim/não), classe econômica pelo critério Brasil 2008 (classe A, B e C), ano de graduação (primeiro ao sexto ano), em função das dimensões QVR, SS e os quatro domínios do WHOQOL: físico (D1), psicológico (D2), relação social (D3) e meio ambiente (D4). Os escores do WHOQOL foram calculados conforme as recomendações da OMS, utilizando sintaxe no SPSS ${ }^{17}$. Para a elaboração das tabelas e gráficos, foi usada também a planilha eletrônica Microsoft Excel. E, para todas as análises, foi considerado o nível de significância $(\alpha)$ de $5 \%{ }^{18}$. Foi realizado teste de diferenças de médias entre renda familiar e as classes do critério Brasil, para verificação da consistência interna do instrumento.

Realizou-se também uma análise estratificada, focando-se apenas no subgrupo com alguma MCR. Para este, foram evidenciados os resultados das médias de QV em cada um dos domínios do WHOQOL segundo as variáveis independentes de análise (sociodemográficas), bem como se realizou o Teste-t e $\mathrm{ANOVA}^{18}$, para comparação das médias em cada domínio para cada variável de interesse.

Esta pesquisa foi aprovada pela Comissão de Ética em Pesquisa do Hospital Universitário Pedro Ernesto (número: 2580; registro CAAE: 0039.0.228000-10), sendo sua realização considerada adequada à população humana. Além disso, todos os participantes (estudantes de medicina) o fizeram voluntariamente, após assinatura do Termo de Consentimento Livre e Esclarecido, onde constava que os dados seriam divulgados apenas coletivamente, garantindo o anonimato dos dados individuais e respeitando a Resolução 196/96 do Conselho Nacional de Saúde.

\section{RESULTADOS}

Em 2010 dos 545 estudantes matriculados na FCM/UERJ, 394 (72\%) participaram da pesquisa, sendo $61 \%$ mulheres e $43 \%$ que ingressaram por cotas (entre estes, $64 \%$ mulheres). O percentual de participantes por ano de graduação foi, respectivamente, de $87,5 \%, 76,6 \%, 67,9 \%, 67,4 \%, 62 \%$ e $74,1 \%$. A idade média foi de 23 anos (16-43a), com diferença estatisticamente significativa quanto à forma de ingresso $(23,5$ x 22,2 anos para cota sim versus cota não, $p=0,000$ ).

Quanto à presença de MCR, 20\% da amostra referiram pelo menos uma. De acordo com a análise por capítulos do CID-10, foi observada a seguinte distribuição de MCR: doenças respiratórias (rinossinusite, bronquite e asma) - 26\%; doenças endócrinas, nutricionais e metabólicas (obesidade, hipotireoidismo e síndrome de ovários policísticos) - 15\%; doenças do aparelho digestivo (gastrite e refluxo gastroesofágico) - 13\%; doenças do aparelho circulatório (hipertensão arterial e varizes); transtornos mentais e comportamentais (ansiedade e depressão); doenças do sistema nervoso (enxaqueca, cefaleia em salvas, epilepsia) e doenças do sistema osteomuscular (tendinite, bursite, hérnia de disco) - 10\% cada uma dessas, perfazendo um total de $94 \%$ das MCR.

Não foi observada diferença nesse percentual considerando-se todas as variáveis de estratificação de interesse: sexo ( $p$ $=0,25)$, forma de ingresso $(p=0,61)$, classe econômica $(p=$ $0,25)$ e ano de graduação $(p=0,32)$. Assim, observou-se, para o sexo masculino, $23 \%$ de MCR e, para o sexo feminino, $18 \%$; para aqueles com forma de ingresso por cotas: sim $(19 \%$ de MCR) e não (21\%). Porém, cabe destacar que, a despeito disso, o perfil de MCR foi diferenciado significativamente para ambos os sexos, em que estudantes do sexo feminino têm OR = 2 para doença mental, e $\mathrm{OR}=1,5$ para doenças endócrino-metabólicas, enquanto estudantes do sexo masculino têm OR = 2 para doenças do aparelho digestivo.

Em relação à escolaridade do chefe da família, 59\% dos estudantes tinham pais com ensino superior completo, sendo a chance de ter o chefe da família sem o nível superior completo cinco vezes maior para os que ingressaram por cotas $(\mathrm{OR}=5,2 ; \mathrm{IC}=3,4-8,1)$. Quanto à renda per capita declarada, observou-se que a dos que ingressaram por cotas foi em média três vezes menor que a declarada pelos não cotistas ( $\mathrm{R} \$ 831,10$ versus $R$ \$ 2.323,60; $p$ valor $=0,000$ ).

Para verificação da consistência interna do instrumento quanto à renda familiar informada, foi realizado teste de diferenças de médias entre estas e as classes econômicas do critério Brasil. A classe A (32\% dos estudantes) referiu renda média familiar R\$11.288,00; a classe B (47\%), R\$4.466,00; e a classe C (21\%), R\$1.996,00, sendo este gradiente decrescente de renda entre as três classes estatisticamente significativo ( $\mathrm{p}$ $<5 \%$ ). Quanto à associação entre a forma de ingresso (cota sim versus cota não) e a classe econômica, detectou-se expressiva diferença entre as proporções de cotistas e não cotistas, principalmente nas classes A e C: classe A ( $8 \%$ versus $50 \%$ ), classe B ( $53 \%$ versus $43 \%$ ) e classe C ( $39 \%$ versus $7 \%)$, com $p=0,000$. 
Na Tabela 1, apresenta-se a análise descritiva da amostra geral dos estudantes, considerando as variáveis de exposição de interesse segundo sua distribuição pelos domínios da QV do WHOQOL-bref. Para a amostra total de estudantes, a média ( \pm desvio padrão) da QVR foi de $66 \pm 23,3$, enquanto que para os domínios esta foi, em ordem decrescente, de $69 \pm 19,5$ para o D3 (relações sociais), $66 \pm 14,4$ para D1 (físico), 63,5 \pm 15,8 para o D2 (psicológico) e $58 \pm 15,8$ para o D4 (meio ambiente).

Observou-se diferença estatisticamente significativa no domínio psicológico (D2) para todas as variáveis investigadas.

Para ambos os sexos, não houve diferença quanto à satisfação com a saúde (SS) e os domínios relações sociais e meio ambiente. Os demais domínios da QV tiveram escores mais baixos para as estudantes em relação aos estudantes, sendo mais expressiva essa diferença para a QVR $(63,4 \pm 23,5$ versus $70,3 \pm 22,3)$ e para o domínio psicológico ( $62 \pm 14,9$ versus 66 $\pm 16,7 ; \mathrm{p}=0,004)$.

Quanto àqueles que referiram ou não morbidade crônica, a diferença mais expressiva foi observada em sua satisfação com a saúde $(50,3 \pm 23$ versus $65 \pm 23,9, \mathrm{p}=0,000)$, não havendo diferença na QVR entre estes estudantes, a despeito daque- les com MCR apresentarem diferenças significativas nos domínios físico, psicológico e relações sociais, em relação àqueles sem MCR (Tabela 1).

Para a forma de ingresso na faculdade, foram observados piores escores de QV para os cotistas, com exceção da satisfação com a saúde. A diferença mais expressiva entre os cotistas e não cotistas se deu no domínio meio ambiente ( $52 \pm 15,7$ versus $63 \pm 14,1 ; \mathrm{p}=0 ; 000)$.

Quanto à classe econômica definida pelo critério Brasil, observou-se um gradiente decrescente em todos os domínios da QV entre as três classes econômicas estatisticamente significativo, sendo marcada essa diferença no domínio meio ambiente (classe $\mathrm{A}=66 \pm 14,5$; classe $\mathrm{B}=57 \pm 12,6$; classe $\mathrm{C}=48 \pm$ $17,5 ; \mathrm{p}=0 ; 000)$ (Tabela 1).

No tocante ao ano de graduação, observou-se um declínio sistemático nos domínios da QV no decorrer do primeiro ao terceiro ano, com recuperação do terceiro ao quinto ano e piora novamente no sexto, chegando para alguns domínios a escores mais baixos do que os encontrados no primeiro ano (Figuras 1 e 2). O domínio meio ambiente se manteve para todos os anos com o mais baixo escore. A QVR cai de um patamar de $73 \pm$

TABELA 1

Escores de qualidade de vida da amostra geral de estudantes de medicina da UERJ, 2010

\begin{tabular}{|c|c|c|c|c|c|c|c|c|}
\hline $\begin{array}{c}\text { Características } \\
\text { investigadas }\end{array}$ & $\mathbf{n}$ & $\%$ & QVR & SS & $\begin{array}{l}\text { D1: } \\
\text { Físico }\end{array}$ & $\begin{array}{l}\text { D2: } \\
\text { Psico }\end{array}$ & $\begin{array}{l}\text { D3: Rel. } \\
\text { Sociais }\end{array}$ & $\begin{array}{l}\text { D4: Meio } \\
\text { ambiente }\end{array}$ \\
\hline \multicolumn{9}{|l|}{ Sexo } \\
\hline Masc. & 154 & 39 & 70,3 & 62,7 & 69,6 & 66,4 & 70,5 & 59,6 \\
\hline Fem. & 240 & 61 & 63,4 & 61,5 & 63,7 & 61,6 & 67,8 & 57,1 \\
\hline Pvalor $(\mathrm{M} / \mathrm{F})$ teste-t & & & 0,004 & 0,633 & 0,000 & 0,004 & 0,186 & 0,127 \\
\hline \multicolumn{9}{|c|}{ Morbidade crônica referida } \\
\hline Sim & 80 & 20 & 62,2 & 50,3 & 58,7 & 57,5 & 62,5 & 55,2 \\
\hline Não & 314 & 80 & 67,1 & 64,9 & 67,9 & 65 & 70,5 & 58,8 \\
\hline Pvalor $(\mathrm{S} / \mathrm{N})$ teste-t & & & 0,090 & 0,000 & 0,000 & 0,001 & 0,003 & 0,072 \\
\hline \multicolumn{9}{|c|}{ Forma de ingresso (cotas) } \\
\hline Sim & 170 & 43 & 61,6 & 60,4 & 63 & 61 & 65,4 & 51,9 \\
\hline Não & 224 & 57 & 69,5 & 63,1 & 68,3 & 65,4 & 71,5 & 62,7 \\
\hline Pvalor $(\mathrm{S} / \mathrm{N})$ teste-t & & & 0,001 & 0,292 & 0,000 & 0,007 & 0,002 & 0,000 \\
\hline \multicolumn{9}{|l|}{ Critério Brasil 2008} \\
\hline Classe A & 127 & 32 & 72,1 & 65,9 & 69,8 & 66,9 & 72,6 & 66,4 \\
\hline Classe B & 186 & 47 & 65,2 & 61,6 & 65,3 & 62,4 & 67,6 & 56,7 \\
\hline Classe C & 81 & 21 & 59,0 & 56,5 & 61,8 & 60,7 & 66,1 & 48,0 \\
\hline Pvalor - Anova & & & 0,000 & 0,023 & 0,000 & 0,008 & 0,026 & 0,000 \\
\hline \multicolumn{9}{|l|}{ Ano de graduação } \\
\hline ano 1 & 70 & 18 & 73,2 & 71,1 & 67,5 & 68,5 & 73,7 & 57,9 \\
\hline ano 2 & 72 & 18 & 70,8 & 59,0 & 65,9 & 64,3 & 68,5 & 58,2 \\
\hline ano 3 & 72 & 18 & 61,5 & 60,4 & 61,9 & 59,5 & 63,5 & 57,7 \\
\hline ano 4 & 60 & 15 & 69,6 & 57,5 & 67,6 & 64,8 & 69,9 & 59,8 \\
\hline ano 5 & 54 & 14 & 64,8 & 63,4 & 69,0 & 64,9 & 73,5 & 59,7 \\
\hline ano 6 & 66 & 17 & 56,4 & 59,9 & 65,2 & 59,4 & 65,4 & 55,5 \\
\hline Pvalor - Anova & & & 0,000 & 0,016 & 0,079 & 0,004 & 0,010 & 0,673 \\
\hline Total geral & 394 & 100 & 66,1 & 61,9 & 66,0 & 63,5 & 68,9 & 58,0 \\
\hline
\end{tabular}


TABELA 2

Escores de qualidade de vida dos estudantes de medicina da UERJ com MCR, 2010

\begin{tabular}{|c|c|c|c|c|c|c|c|c|}
\hline $\begin{array}{c}\text { Características } \\
\text { investigadas }\end{array}$ & $\mathbf{n}$ & $\%$ & QVR & SS & $\begin{array}{l}\text { D1: } \\
\text { Físico }\end{array}$ & $\begin{array}{l}\text { D2: } \\
\text { Psico }\end{array}$ & $\begin{array}{l}\text { D3: Rel. } \\
\text { Sociais }\end{array}$ & $\begin{array}{l}\text { D4: Meio } \\
\text { ambiente }\end{array}$ \\
\hline \multicolumn{9}{|l|}{ Sexo } \\
\hline Masc. & 36 & 45 & 67,4 & 52,8 & 69,6 & 66,4 & 70,5 & 59,6 \\
\hline Fem. & 44 & 55 & 58,0 & 48,3 & 63,7 & 61,6 & 67,8 & 57,1 \\
\hline Pvalor $(\mathrm{M} / \mathrm{F})$ teste-t & & & 0,080 & 0,390 & 0,000 & 0,004 & 0,186 & 0,127 \\
\hline \multicolumn{9}{|l|}{ Forma de ingresso (cota) } \\
\hline Sim & 32 & 40 & 53,9 & 46,1 & 54,2 & 54,6 & 56,6 & 46,0 \\
\hline Não & 48 & 60 & 67,7 & 53,0 & 61,7 & 59,6 & 66,5 & 61,4 \\
\hline Pvalor $(\mathrm{S} / \mathrm{N})$ teste-t & & & 0,009 & 0,189 & 0,047 & 0,231 & 0,047 & 0,000 \\
\hline \multicolumn{9}{|l|}{ Critério Brasil 2008} \\
\hline Classe A & 28 & 35 & 74,1 & 57,1 & 65,3 & 62,8 & 71,1 & 66,2 \\
\hline Classe B & 40 & 50 & 56,3 & 46,9 & 55,8 & 53,8 & 59,4 & 51,9 \\
\hline Classe C & 12 & 15 & 54,2 & 45,8 & 53,0 & 58,0 & 52,8 & 40,7 \\
\hline Pvalor - Anova & & & 0,004 & 0,149 & 0,025 & 0,129 & 0,022 & 0,000 \\
\hline \multicolumn{9}{|l|}{ Ano de graduação } \\
\hline ano 1 & 8 & 10 & 78,1 & 62,5 & 66,1 & 67,7 & 62,5 & 56,8 \\
\hline ano 2 & 22 & 27 & 68,2 & 45,5 & 56,8 & 58,7 & 63,6 & 54,6 \\
\hline ano 3 & 13 & 16 & 61,5 & 50,0 & 55,5 & 54,5 & 54,5 & 59,5 \\
\hline ano 4 & 11 & 14 & 63,6 & 54,6 & 59,1 & 55,3 & 60,6 & 58,5 \\
\hline ano 5 & 6 & 8 & 62,5 & 62,5 & 70,2 & 74,3 & 81,9 & 71,5 \\
\hline ano 6 & 20 & 25 & 48,8 & 45,0 & 56,3 & 50,4 & 61,7 & 45,8 \\
\hline Pvalor - Anova & & & 0,045 & 0,277 & 0,332 & 0,040 & 0,259 & 0,029 \\
\hline Total com MCR & 80 & 100 & 62,2 & 50,3 & 58,7 & 57,5 & 62,5 & 55,2 \\
\hline
\end{tabular}

FigURA 1

Qualidade de vida referida pelos estudantes de medicina e sua satisfação com a saúde pelo WHOQOL-bref, segundo ano de graduação. UERJ, 2010.

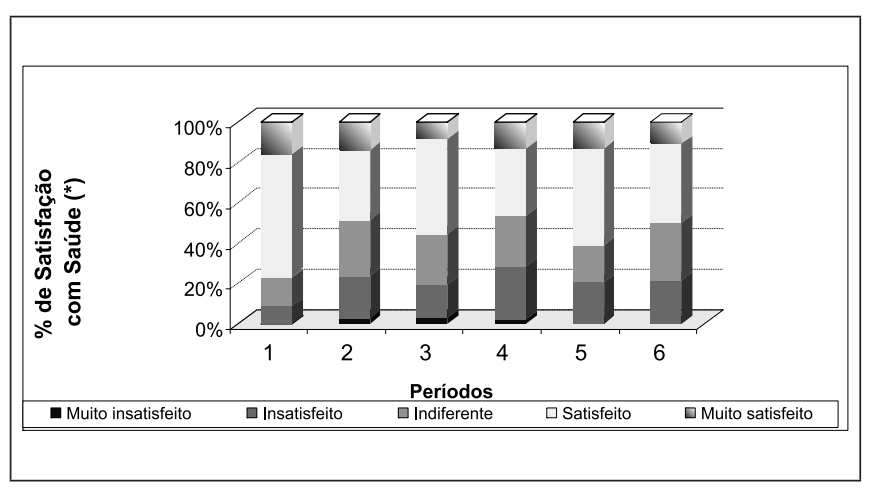

(*) $p$-valor do qui-quadrado $=0,056$

19,2 , no primeiro ano, para $56 \pm 22,9 ; \mathrm{p}=0 ; 000$, no sexto ano, e a SS cai de $71 \pm 19,8$ para $60 \pm 23,6 ; p=0,016$ (Tabela 1 ).

Na tabela 2 destacam-se os resultados relativos especificamente aos estudantes com MCR que, como exposto anteriormente, apresentaram menores escores médios em todos os do-
Figura 2

Escores de qualidade de vida (WHOQOL-bref) por ano de graduação de estudantes de medicina. UERJ, 2010.

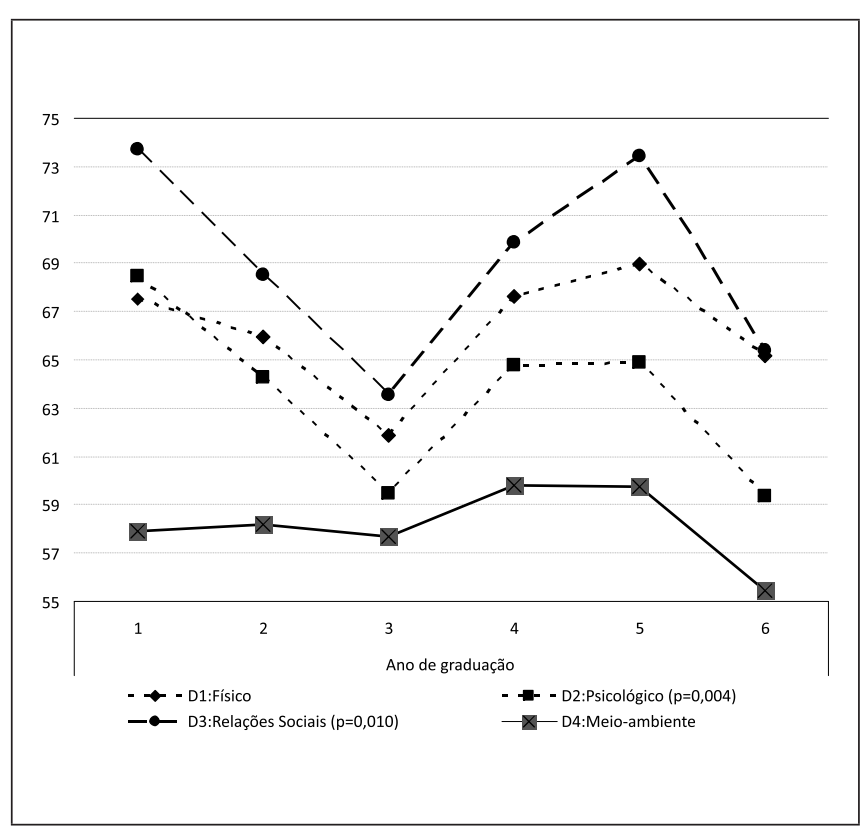


mínios do WHOQOL-bref. Dentro do grupo dos 80 estudantes com MCR houve diferença estatisticamente significativa para os alguns domínios da QV de acordo com a variável estudada, mantendo certa semelhança com os resultados obtidos na amostra geral. Desse modo, os domínios físico e psicológico foram menores para as mulheres em relação aos homens; a QVR e os domínios físico, relações sociais e meio ambiente foram menores para os cotistas e classe $C$, mas aí já não se observa mais diferença no domínio psicológico; e, QVR e os domínios psicológico e meio ambiente foram menores para os estudantes do sexto ano. A SS, contudo, não diferiu de acordo com o ano, sendo o valor médio encontrado de 50.

\section{DISCUSSÃO}

A saúde, principalmente mental, dos estudantes de medicina há muito tem sido objeto de atenção e pesquisas em nosso meio $^{19,20}$. O estresse e problemas mentais, como a ansiedade e a depressão, são descritos nessa população de estudantes e associados ao baixo rendimento acadêmico ${ }^{21}$. A carga horária dos estudantes na faculdade é extensa, distribuída em atividades de ensino, pesquisa e extensão. $\mathrm{O}$ estresse decorre da dificuldade em administrar o tempo exigido pelas diferentes disciplinas e o lazer. Soma-se a isso o contato com os pacientes e com a morte, bem como a competitividade entre os colegas, que se acirra com a valorização do desempenho acadêmico durante a graduação, perdurando até o processo seletivo para a entrada na residência.

Embora encontremos na literatura dados sobre a presença de doenças crônicas em estudantes de medicina ${ }^{22,23}$, chama a atenção, em nossa amostra, a prevalência de $20 \%$ de MCR em jovens com idade média de 23 anos e o fato de muitas dessas morbidades crônicas poderem ser resultado direto de estresse ou por ele exacerbadas.

Como observado em uma pesquisa realizada na população geral na região Sul do nosso país ${ }^{24}$ o grupo que referia morbidade crônica apresentou menores escores de QV nos domínios aferidos pelo WHOQOL-bref. Para os estudantes com MCR, não houve diferença estatística significativa na satisfação com a saúde para os diferentes estratos das variáveis analisadas, mas para a QVR houve (Tabela 2), tal como observado para a amostra geral (Tabela 1), o que ratifica a QV como um constructo mais abrangente que a saúde física. Dito de outra forma, controlando o fator de confundimento "ter MCR", ainda assim a QV dos estudantes foi significativamente influenciada pelas características socioeconômicas sob análise.

As diferenças encontradas neste estudo com relação ao sexo vão ao encontro dos dados da literatura sobre estudantes de medicina ${ }^{10,11}$ e sobre a população geral, que revelam pior QVR para mulheres ${ }^{24}$ e maior vulnerabilidade destas aos sintomas ansiosos e depressivos em relação aos homens ${ }^{25,26,27}$, assim como maior prevalência de doenças endócrino-metabólicas em estudantes do sexo feminino ${ }^{22}$.

O sistema de cotas para ingresso nas universidades estaduais é regido pela Lei Estadual sobre a Reserva de Vagas $5.346 / 08$, que estabelece $45 \%$ de vagas para ingresso de estudantes com menor poder aquisitivo nas universidades públicas estaduais do Rio de Janeiro, com vistas à redução de desigualdades étnicas, sociais e econômicas, sendo o critério de limite de renda bruta média familiar na UERJ de R $\$ 960,00$ desde $2009^{28}$.

Diferentemente do que acontece em outros cursos de graduação oferecidos pela UERJ, em que o número de estudantes matriculados através da reserva de vagas tem decrescido ${ }^{29}$, na medicina, as vagas vêm sendo de fato preenchidas por estudantes que atendem ao critério socioeconômico estabelecido no edital, dado que a renda média per capita declarada pelos estudantes cotistas (43\% desta amostra) foi de $\mathrm{R} \$ 831,00$.

O cumprimento a esta lei já se reflete no perfil do estudante de medicina da UERJ. Em 1996, 82\% dos estudantes do primeiro ano da FCM tinham pais graduados e 55\% tinha parentes médicos ${ }^{30}$. Em 2010, como mencionado, o percentual de estudantes com pais graduados caiu para 59\%, dados próximos aos encontrados no censo de 2011 dessa faculdade, que foi de $62 \%$. Ainda nesse censo, $60 \%$ dos estudantes negaram parentesco com médicos ${ }^{31}$.

A QV dos estudantes que ingressaram por cota foi mais baixa que a observada na amostra geral, sendo que as maiores diferenças se deram nos domínios relações sociais (D3) e meio ambiente (D4). Como não foi investigado o local de moradia, o acesso a bens culturais ou às redes sociais de apoio dos estudantes, não se pode inferir sobre as relações entre as diferentes formas de capital (econômico, cultural e social) nessa amostra, a despeito do que Bourdieu aponta em sua obra A distinção: crítica social do julgamento ${ }^{32}$.

O D4 (meio ambiente) foi sempre o mais baixo em todas as características sociodemográficas pesquisadas. A literatura aponta que esse domínio, que trata de questões como segurança, recursos financeiros, transporte e outros, é muito mais sensível às variações socioeconômicas que os demais ${ }^{33}$.

Como a carga horária do curso de medicina é extensa, considera-se quase que "mandatória" a dedicação exclusiva do estudante, de modo que a formação profissional depende das condições objetivas de existência das famílias ${ }^{34}$. Em estudo anteriormente realizado na UERJ ${ }^{30}, 85 \%$ dos egressos do curso de medicina eram economicamente dependentes dos pais. $\mathrm{Na}$ amostra do presente estudo, não foi investigado o exercício de trabalho remunerado por parte dos estudantes, porém pesquisa realizada na Escola de Medicina da Bahia indicou que isso 
pode ser protetor para os estudantes de medicina em relação à presença de transtornos mentais comuns ${ }^{35}$.

Para ampliar as possibilidades dos cotistas permanecerem na universidade até a conclusão do curso, a UERJ criou o Programa de Iniciação Acadêmica (PROINICIAR) que oferece: bolsa permanência - atualmente no valor de $\mathrm{R} \$ 400,00$; material didático (escolhido pela direção das unidades acadêmicas); bilhete único carioca — instituído pela Lei Municipal 5266/2011; atividades acadêmicas opcionais, entre outros serviços para atendimento do estudante que ingressa pelo sistema de $\operatorname{cotas}^{36}$.

A hipótese de que os seis anos do curso médico afetam negativamente a QV dos estudantes foi demonstrada anteriormente $^{11,12,37}$ e ratificada aqui pelo gradiente decrescente verificado nos domínios do WHOQOL ao longo dos seis anos da graduação. Contudo, não se repetiu aqui a melhora no D3 (relações sociais) no percurso da formação médica, fato observado em outros estudos em que o poder aquisitivo dos estudantes ou não foi avaliado ${ }^{11}$ ou era alto ${ }^{23}$.

Em nossa amostra houve uma queda dos escores dos domínios físico, psicológico e relações sociais no terceiro ano e novamente no sexto ano (Figura 2). No terceiro ano acontece a transição do básico para a clínica, com a entrada no hospital e o ensino-aprendizagem de semiologia. É chegada a hora dos estudantes começarem a aprender (ou não) a lidar com os processos de adoecimento e morte dos pacientes ${ }^{38}$. O sexto ano é o momento de enfrentar as expectativas com a formatura, a escolha da especialidade e o ingresso na vida profissional. Estudo realizado na China, onde, a despeito de durar cinco anos o curso médico guarda semelhanças com o da FCM/UERJ, também revelou piores escores para todos os domínios no terceiro ano ${ }^{39}$.

Esses são exemplos de crises pelas quais os estudantes de medicina passam no decorrer da graduação, cujos meios para a adaptação ou superação parecem ainda depender muito mais de características pessoais na forma de lidar com o estresse e de gerenciar o tempo para conciliar os estudos com as outras dimensões da vida ${ }^{13,40}$.

Entretanto, a Escola Médica deve refletir sobre o seu papel no acolhimento e acompanhamento dos estudantes ao longo do seu processo de formação, de modo que a valorização da dimensão psicológica e o cuidado oferecido aos estudantes não fiquem a cargo apenas dos serviços de apoio psicopedagógico ou grupos de tutoria, mas façam parte do encontro entre professores e alunos nos diferentes cenários de ensino-aprendizagem.

A avaliação de uma experiência americana sobre a oferta de uma disciplina eletiva visando à promoção do bem-estar dos estudantes revelou uma boa aceitação por aqueles que a frequentaram, porque aprenderam a perceber e a lidar melhor com o estresse ${ }^{41}$. Em outra pesquisa, estudantes que afirma- ram que sua escola tentava minimizar o estresse, ou apresentava um bom sistema para lidar com este, referiram menos dias com o humor deprimido ${ }^{42}$.

No presente estudo, utilizou-se um instrumento validado internacionalmente, inclusive no Brasil (Whoqol-bref), além disso, obteve-se uma elevada representatividade dos estudantes matriculados em 2010 (em torno de 75\% em cada ano de graduação), com importante cobertura de resposta em cada questionário ("completitude" de cerca de $98 \%$ das perguntas respondidas), o que minimiza a possibilidade de viés de seleção, de voluntário e de aferição; mas, ainda assim, este estudo pode ter limitações metodológicas.

Em primeiro lugar, entende-se que um desenho de coorte prospectivo durante os seis anos do curso médico seria mais apropriado para acompanhar a trajetória de um mesmo grupo e conhecer as mudanças em sua QV no decorrer da graduação, mas optou-se por um estudo transversal em função de questões operacionais e recursos disponíveis. Nesse sentido, fica a sugestão para pesquisas futuras.

Em segundo, embora se tenha procurado controlar o confundimento nos resultados da QV por meio da realização de análises estratificadas pelas diversas variáveis socioeconômicas, outras importantes variáveis que poderiam influenciar a QV não foram avaliadas, tais como: local de moradia, tempo e forma de deslocamento da residência à faculdade, exercício de trabalho remunerado, satisfação com o curso, resiliência, entre outras.

O estudo exploratório aqui apresentado está sendo complementado com um estudo multivariado, a fim de se controlarem possíveis confundimentos e/ou interações entre as variáveis de exposição, aplicando-se análise de regressão linear múltipla, tendo como desfechos os quatro domínios do WHOQOL, a partir da base de dados quantitativa em questão.

Como outra limitação, tem-se que o presente estudo foi de caráter apenas quantitativo, sem possibilidade de investigação e checagem em profundidade acerca de questionamentos suscitados, como a compreensão sobre as percepções dos alunos sobre qualidade de vida e saúde e sobre a relação entre a sua qualidade de vida e o percurso da formação médica.

\section{CONCLUSÕES}

Observou-se neste estudo significativa diferença na QV para os estudantes do sexo feminino, dos que apresentavam morbidade crônica, dos que ingressaram através do sistema de reserva de vagas, dos provenientes da classe econômica $\mathrm{C}$ e dos que estão nos terceiro e sexto anos da graduação. Os achados ratificam a consistência interna do instrumento e apontam para a necessidade de estudos qualitativos para a compreensão da percepção dos estudantes sobre a sua QV. 


\section{AGRADECIMENTOS}

Aos estudantes de medicina da UERJ que participaram da pesquisa e ao digitador Silvio Rodrigues Campos.

\section{REFERÊNCIAS}

1. Fleck MP. Problemas conceituais em qualidade de vida. In:Fleck MP. A avaliação de qualidade de vida. Guia para profissionais de saúde. Porto Alegre: Artmed; 2008. p. 19-28.

2. Engel GL. The need for a new medical model: a challenge for biomedicine. Science 1977;196:129-36.

3. Balint M. O médico, seu paciente e a doença. Rio de Janeiro: Atheneu; 1975.

4. Ventegodt S, Merrick J, Anderson NJ. Quality of Life Theory I. The IQOL Theory: an Integrative Theory of the Global Quality of Life Concept. Scientific World Journal. 2003;3:1030-40.

5. Minayo MCS, Hartz ZM, Buss PM. Qualidade de vida e saúde: um debate necessário. Ciênc Saúde Coletiva. 2000;5(1):7-18.

6. The Whoqol Group. The World Health Organization quality of life assessment (WHOQOL): position paper from the World Health Organization. Soc Sci Med.1995;10:1403-9.

7. Fleck MP, Leal OF, Louzada S, Xavier M, Chachamovich E, Vieira G, et al. Desenvolvimento da versão em português do instrumento de avaliação de qualidade de vida da OMS (WHOQOL-100). Rev Bras Psiquiatr. 1999;21(1):1928. DOI: $10.1590 /$ S1516-44461999000100006.

8. Chachamovich E, Fleck MP. Desenvolvimento do WHOQOL-BREF. In: Fleck MP. A avaliação de qualidade de vida. Guia para profissionais de saúde. Porto Alegre: Artmed; 2008. p.74-82.

9. Kluthcovsky AC, Kluthcovsky FA. WHOQOL-bref, um instrumento para avaliar qualidade de vida: uma revisão sistemática. Rev Psiquiat Rio Gd Sul [periódico na internet]. 2009 [acesso em 22 abr. 2012]; 31(3 Supl.). Disponível em: http:/ /dx.doi.org/10.1590/S0101-81082009000400007.

10. Fiedler PT. Avaliação da qualidade de vida do estudante de medicina e da influência exercida pela formação acadêmica. São Paulo; 2008. Doutorado [Tese] - Faculdade de Medicina da Universidade de São Paulo.

11. Alves JGB, Tenório M, Anjos AG, Figueroa JN. Qualidade de vida em estudantes de medicina no início e final do curso: avaliação pelo Whoqol-bref. Rev Bras Educ Med. 2010;34(1):91-6. DOI: 10.1590/S0100-55022010000100011

12. Ramos-Dias JC, Libardi MC, Zillo CM, Igarashi MH, Senger $\mathrm{MH}$. Qualidade de vida em cem alunos do curso de Medicina de Sorocaba - PUC/SP. Rev Bras Educ Med. 2010;34(1):116-23. DOI: 10.1590/S0100-55022010000100014
13. Pereira PB. Bem-estar e busca de ajuda: um estudo junto a alunos de medicina ao final do curso. São Paulo; 2010. Mestrado [Dissertação] - Faculdade de Medicina da Universidade de São Paulo.

14. Krägeloh CU, Henning MA, Hawken SJ, Zhao Y, Shepherd D, Billington R. Validation of the WHOQOL-BREF Quality of Life Questionnaire for Use with Medical Students. Educ Health (Abingdon) [periodico na internet]. 2001 [acesso em 10 jul. 2012]; 24(2):1-5. Disponível em: http://www. educationforhealth.net/

15. Moreno AB, Faerstein E, Werneck GL, Lopes CS, Chor D. Propriedades psicométricas do Instrumento Abreviado de Avaliação de Qualidade de Vida da Organização Mundial da Saúde no Estudo Pró-Saúde. Cad Saúde Pública. 2006;22(12):2585-97. DOI: 10.1590/S0102-311 X2006001200009

16. Associação Brasileira de Empresas de Pesquisa. Critério de classificação econômica Brasil: dados com base no levantamento socioeconômico 2005 IBOPE [on-line]. 2008. [acesso em 3 maio 2012]. Disponível em: http: / / www.google.com/url?q=http:/ / www.abep. org/novo/FileGenerate.ashx\%3Fid\%3D250\&sa=U\&ei=sYaiT9vsLKS16AHNsY30CA\&ved=0CA0QFjAE\&cli ent=internal-uds-cse\&usg=AFQjCNF_9NTuOD6bfhX-L_ 6z4TsAgsNOPw

17. Sintaxe SPSS. WHOQOL-bref Questionnaire. [acesso em 10 maio 2012]. Disponível em: http://www.ufrgs.br/ psiq/whoqol86.html

18. Barbetta PA. Estatística Aplicada às Ciências Sociais. $7^{\underline{a}}$ ed. Florianópolis: EdUfsc; 2011.

19. Millan RL. A assistência psicológica ao estudante de medicina no Brasil: notas históricas. In: Millan LR, De Marco OL, Rossi E. et al. O universo psicológico do futuro médico: vocação, vicissitudes e perspectivas. São Paulo: Casa do Psicólogo; 1999. p.245-82.

20. Guimarães KB (org.). A saúde mental do médico e do estudante de medicina. São Paulo: Casa do Psicólogo; 2007.

21. Stewart SM, Lam TH, Betson CL, Wong CM, Wong AM. A prospective analysis of stress and academic performance in the first two years of medical school. Med Educ. 1999;33(4):243-50.

22. Conte M, Gonçalves A. Ampliando elementos da educação médica: morbidade referida em universitários de medicina. Rev Bras Educ Med. 2006;30(1):15-9. DOI: 10.1590/ S0100-55022006000100003

23. Dias JC, Libardi MC, Zillo CM. Qualidade de vida em cem alunos do curso de medicina de Sorocaba - PUC/SP. Rev Bras Educ Med. 2010;34(1):116-23. DOI: 10.1590/S010055022010000100014 
24. Cruz LN, Polanczyk CA, Camey AS, Hoffmann JF, Fleck MP. Quality of life in Brazil: normative values for the Whoqol-bref in a southern general population sample. Qual Life Res. 2011;20:1123-9. DOI: 10.1007/s11136-011-9845-3

25. Arôca SR. Qualidade de vida: comparação entre o impacto de ter transtorno mental comum e a representação do sofrimento dos nervos em mulheres. Rio de Janeiro; 2009. Mestrado [Dissertação]- Escola Nacional de Saúde Pública Sergio Arouca.

26. Andrade LH, Viana MC, Silveira CM. Epidemiologia dos transtornos psiquiátricos na mulher. Rev Psiquiatr Clín. 2006;33(2):45-54.

27. Fortes, S, Villano, LA, Lopes, CS. Nosological profile and prevalence of common mental disorders of patients seen at the Family Health Program (FHP) units in Petrópolis, Rio de Janeiro. Rev Bras Psiquiatr 2008;30(1):32-7.

28. Universidade do Estado do Rio de Janeiro. Vestibular UERJ. Instruções específicas para os candidatos às vagas do sistema de cotas [online]. [acesso em 23 maio 2013]. Disponível em: http://www.vestibular.uerj.br/portal_vestibular_uerj/arquivos/arquivos2009/Anexo_3_ED.pdf

29. Amadei S. Sistema de cotas na UERJ: uma análise quantitativa dos dados de ingresso. Rev Eletrônica Vest. [periódico na internet]. 2008 [acesso em 16 maio 2012]. Disponível em: http://www.revista.vestibular.uerj.br/artigo/artigo. php?seq_artigo $=6$

30. Costa-Macedo LM , Taquette SR, Alvarenga FB. Da adolescência à maturidade: estudo prospectivo dos estudantes de medicina da Universidade do Estado do Rio de Janeiro. Rev Bras Educ Med 2003 jan./abr;27(1):29-35.

31. Aranha RN, Machado APG, Rangel M. Censo MedUERJ 2011. Apresentação dos resultados preliminares no III Seminário de Aprimoramento Curricular promovido pela FCM/UERJ em dezembro de 2012

32. Alves ER. Pierre Bourdieu: a distinção de um legado de práticas e valores culturais. Soc Estado. 2008 [acesso em 25 jun. 2012];23(1):179-84. Disponível em: http://www.scielo.br/pdf/se/v23n1/a09v23n1.pdf

33. Gordia AP, Quadros TM, Campos W. Variáveis sociodemográficas como determinantes do domínio meio ambiente da qualidade de vida de adolescentes. Ciênc Saúde Coletiva. $2009 ; 14(6): 2261-8$

34. Nogueira MA. A construção da excelência escolar: um estudo de trajetórias feito com estudantes universitários provenientes das camadas médias intelectualizadas. In: Nogueira MA, Romanelli G, Zago N, (org.). Família e escola: trajetórias de escolarização em camadas médias e populares. Petrópolis, RJ: Vozes; 2000. p.15-43.
35. Almeida AM, Godinho TM, Bitencourt AGV, Teles MS, Silva AS, Fonseca DC et al. Common mental disorders among medical students. J Bras Psiquiatr. 2007;56(4):245-51. DOI: 10.1590/S0047-20852007000400002

36. Programa de Iniciação Acadêmica [homepage].[acesso em 14 jun. 2012]. Disponível em: http://www.proiniciar.uerj. br/

37. Paro HBM, Morales NM, Silva $\mathrm{CH}$, Rezende $\mathrm{CH}$, Pinto RM, Morales RR, et al. Health-related quality of life of medical students. Med Educ. 2010;44(3):227-35. DOI: 10.1111/j.1365-2923.2009.03587.x.

38. Azeredo NSG, Rocha CF, Carvalho PRA. O enfrentamento da morte e do morrer na formação de acadêmicos de medicina. Rev Bras Educ Med. 2011;35(1):37-43. DOI: 10.1590/ S0100-55022011000100006

39. Zhang Y, Qu B, Lun S, Wang D, Guo Y, Liu J. Quality of Life of Medical Students in China: A Study Using the WHOQOL-BREF. PLoS ONE 2012; 7(11): e49714. doi:10.1371/ journal.pone.0049714

40. Zonta R, Robles ACC, Grosseman S. Estratégias de enfrentamento do estresse desenvolvidas por estudantes de medicina da Universidade Federal de Santa Catarina. Rev Bras Educ Med. 2006;30(3):147-53. DOI: 10.1590/S010055022006000300005.

41. Lee J, Graham AV. Students' perception of medical school stress and their evaluation of a wellness elective. Med Educ. 2001;35:652-9.

42. Compton MT, Carrera J, Frank E. Stress and Depressive Symptoms/Dysphoria Among US Medical Students Results From a Large, Nationally Representative Survey. J Nerv Ment Dis.2008;196(12): 391-7.

\section{CONTRIBUIÇÃO DOS AUTORES}

Ambas participaram da concepção e projeto ou análise e interpretação dos dados, da redação do artigo ou revisão crítica relevante do conteúdo intelectual e da aprovação final da versão a ser publicada.

\section{CONFLITO DE INTERESSES}

Declarou não haver.

\section{ENDEREÇO PARA CORRESPONDÊNCIA}

Ana Claudia Santos Chazan

Rua Pereira da Silva, 586 - apto. 006

Laranjeiras - Rio de Janeiro

CEP. 22221-140 RJ

E-mail: anachazan@gmail.com 